\title{
Glucose toxicity and the development of diabetes in mice with muscle-specific inactivation of GLUT4
}

\author{
Jason K. Kim, ${ }^{1,2}$ Ariel Zisman, ${ }^{3}$ Jonathan J. Fillmore, ${ }^{2}$ Odile D. Peroni, ${ }^{4}$ Ko Kotani, ${ }^{4}$ \\ Pascale Perret, ${ }^{2}$ Haihong Zong, ${ }^{2}$ Jianying Dong, ${ }^{2}$ C. Ronald Kahn, ${ }^{3}$ Barbara B. Kahn, ${ }^{4}$ \\ and Gerald I. Shulman ${ }^{1,2}$
}

\author{
${ }^{1}$ Howard Hughes Medical Institute, and \\ ${ }^{2}$ Department of Internal Medicine, Yale University School of Medicine, New Haven, Connecticut, USA \\ 3Joslin Diabetes Center, Department of Medicine, and \\ ${ }^{4}$ Department of Medicine, Beth Israel Deaconess Medical Center, Harvard Medical School, Boston, Massachusetts, USA
}

Address correspondence to: Gerald I. Shulman, Howard Hughes Medical Institute, Yale University School of Medicine, Boyer Center for Molecular Medicine, 295 Congress Avenue, Boyer Center for Molecular Medicine, Room 254C, Box 9812, New Haven, Connecticut 06536-8012, USA. Phone: (203) 737-1115; Fax: (203) 737-4059; E-mail: gerald.shulman@yale.edu.

Received for publication May 10, 2000, and accepted in revised form May 14, 2001.

\begin{abstract}
Using cre/loxP gene targeting, transgenic mice with muscle-specific inactivation of the GLUT4 gene (muscle GLUT4 KO) were generated and shown to develop a diabetes phenotype. To determine the mechanism, we examined insulin-stimulated glucose uptake and metabolism during hyperinsulinemic-euglycemic clamp in control and muscle GLUT4 KO mice before and after development of diabetes. Insulin-stimulated whole body glucose uptake was decreased by $55 \%$ in muscle GLUT4 KO mice, an effect that could be attributed to a $92 \%$ decrease in insulin-stimulated muscle glucose uptake. Surprisingly, insulin's ability to stimulate adipose tissue glucose uptake and suppress hepatic glucose production was significantly impaired in muscle GLUT4 KO mice. To address whether these latter changes were caused by glucose toxicity, we treated muscle GLUT4 KO mice with phloridzin to prevent hyperglycemia and found that insulin-stimulated whole body and skeletal muscle glucose uptake were decreased substantially, whereas insulin-stimulated glucose uptake in adipose tissue and suppression of hepatic glucose production were normal after phloridzin treatment. In conclusion, these findings demonstrate that a primary defect in muscle glucose transport can lead to secondary defects in insulin action in adipose tissue and liver due to glucose toxicity. These secondary defects contribute to insulin resistance and to the development of diabetes.
\end{abstract}

J. Clin. Invest. 108:153-160 (2001). DOI:10.1172/JCI200110294.

\section{Introduction}

Although it is widely accepted that insulin resistance is a major factor in the pathogenesis of type 2 diabetes $(1$, $2)$, the precise mechanism by which insulin resistance leads to the development of type 2 diabetes is uncertain. Furthermore, patients with type 2 diabetes exhibit insulin resistance in multiple organs (i.e., skeletal muscle, adipose tissue, and liver) (3), and it is unknown whether a defect in one or all of these organs is required for the development of diabetes. Skeletal muscle is responsible for the majority of whole body insulinmediated glucose metabolism $(4,5)$, and insulin promotes glucose transport in this tissue by stimulating the translocation of the major insulin-responsive glucose transporter, GLUT4, from intracellular storage vesicles to the plasma membrane (6-8). Decreased insulin-stimulated glucose transport in skeletal muscle has been shown to be a major contributing factor to the insulin resistance in patients with type 2 diabetes $(9,10)$ and obesity (11). Recently, Zisman and colleagues (12) developed mice with muscle-specific disruption of the GLUT4 gene (muscle GLUT4 knockout $[\mathrm{KO}]$ mice), and these mice develop glucose intolerance and a diabetes phenotype. To examine the mechanism by which diabetes develops in these mice, we performed hyperinsulinemic-euglycemic clamps in awake muscle GLUT4 KO mice before and after the development of diabetes. Moreover, in order to examine whether prolonged hyperglycemia (i.e., glucose toxicity) may be responsible for the development of diabetes in muscle GLUT4 KO mice, we chronically treated muscle GLUT4 KO mice with phloridzin, which inhibits renal glucose reabsorption (13), to prevent hyperglycemia and examined insulin action in vivo.

\section{Methods}

Animals. Male control (wild-type, lox/lox, and cre; $n=5-10$ for each group), heterozygous $\mathrm{KO}$ (cre +/lox; $n=8)$, and muscle GLUT4 KO (12) $(n=8-11$ for each group) littermates were studied at approximately 10 (young) and 20-21 weeks of age. For the phloridzintreated (PHZ-treated) muscle GLUT4 KO group $(n=5)$, PHZ (0.7 mg/day; Sigma Chemical Co., St. Louis, Missouri, USA) was chronically administered in muscle GLUT4 KO mice from 12 to 20 weeks of age using an osmotic pump. Animals were housed under controlled 
temperature $\left(23^{\circ} \mathrm{C}\right)$ and lighting (12 hours of light, 0600-1800 hours; 12 hours of dark, $1800-0600$ hours) with free access to water and standard mouse chow. All procedures were approved by the Yale University Animal Care and Use Committee.

Surgery and animal handling. For the chronic PHZ treatment, mice were anesthetized with an intraperitoneal injection of ketamine $(100 \mathrm{mg} / \mathrm{kg}$ body weight $)$ and xylazine $(10 \mathrm{mg} / \mathrm{kg}$ body weight), and an Alzet osmotic pump (Alza Corp., Palo Alto, California, USA) containing PHZ (9.3 $\mathrm{mg}$ in $3 \mathrm{ml}$ saline) was implanted subcutaneously. At least 4 days before experiments, all mice were anesthetized with ketamine and xylazine, and an indwelling catheter was inserted in the left internal jugular vein. The catheters were externalized through an incision in the skin flap behind the head, and the mice were returned to individual cages after the surgery. The mice were fully recuperated from the surgery before the in vivo experiments, as reflected by their reaching preoperative weight. To conduct experiments in awake animals with minimal stress, a tail-restraint method was used (14).

Hyperinsulinemic-englycemic clamp. Experiments were started at 1000 hours after an overnight fast (food was removed at 1700 hours on the day before the experiment). A 120-minute hyperinsulinemic-euglycemic clamp was conducted with a prime-continuous infusion of human insulin (Eli Lilly and Co., Indianapolis, Indiana, USA) at a rate of $15 \mathrm{pmol} / \mathrm{kg} / \mathrm{min}$ to raise plasma insulin within a physiological range $(780 \mathrm{pM})$. Blood samples $(20 \mu \mathrm{l})$ were collected at $20-$ to $30-$ minute intervals for the immediate measurement of plasma glucose concentration, and $20 \%$ glucose was infused at variable rates to maintain plasma glucose at basal concentrations. Insulin-stimulated whole body glucose flux was estimated using a prime-continuous infusion of HPLC-purified [ $\left.3-{ }^{3} \mathrm{H}\right]$ glucose $(10 \mu \mathrm{Ci}$ bolus, $0.1 \mu \mathrm{Ci} / \mathrm{min}$; NEN Life Science Products Inc., Boston, Massachusetts, USA) throughout the clamps. To estimate the rate of basal glucose turnover, $\left[3-{ }^{3} \mathrm{H}\right]$ glucose $(0.05 \mu \mathrm{Ci} / \mathrm{min})$ was infused for 2 hours (basal period) before the start of clamps, and a blood sample $(20 \mu \mathrm{l})$ was taken at the end of this basal period. Rates of insulin-stimulated glucose uptake in individual tissues in vivo were estimated using 2 -deoxy-D-[1-14 C]glucose $\left(2-\left[{ }^{14} \mathrm{C}\right] \mathrm{DG}\right)$, a nonmetabolizable glucose analogue and by determining the tissue content of 2-deoxyglucose-6phosphate. To estimate insulin-stimulated glucose uptake and metabolism in individual tissues, 2$\left[{ }^{14} \mathrm{C}\right] \mathrm{DG}$ (NEN Life Science Products Inc.) was administered as a bolus $(10 \mu \mathrm{Ci})$ at 45 minutes before the end of the clamps. Blood samples $(20 \mu \mathrm{l})$ were taken at 80 , $85,90,100,110$, and 120 minutes after the start of the clamps for the determination of plasma $\left[{ }^{3} \mathrm{H}\right]$ glucose, ${ }^{3} \mathrm{H}_{2} \mathrm{O}$, and $2-\left[{ }^{14} \mathrm{C}\right]$ DG concentrations. Additional blood samples $(20 \mu \mathrm{l})$ were collected before the start and at the end of the clamps for measurement of plasma insulin concentrations. All infusions were done using microdialysis pumps (CMA/Microdialysis, North Chelmsford, Massachusetts, USA). At the end of clamps, animals were anesthetized with sodium pentobarbital injection $(2 \mathrm{mg} / \mathrm{kg}$ body weight). Within 5 minutes, four muscles (soleus, gastrocnemius, tibialis anterior, and quadriceps) from both hindlimbs, epididymal white adipose tissue, intrascapular brown adipose tissue, and liver were taken. Each tissue, once exposed, was dissected out within 2 seconds, frozen immediately using liquid $\mathrm{N}_{2}$-cooled aluminum blocks, and stored at $-70^{\circ} \mathrm{C}$ for later analysis.

In vivo glucose-flux analysis. Plasma glucose during clamps was analyzed using $10 \mu \mathrm{l}$ plasma by a glucose oxidase method on a Beckman glucose analyzer 2 (Beckman Instruments Inc., Fullerton, California, USA). Plasma insulin was measured by RIA using kits from Linco Research Inc. (St. Charles, Missouri, USA). For the determination of plasma $\left[3-{ }^{3} \mathrm{H}\right]$ glucose and 2-[ $\left[{ }^{14} \mathrm{C}\right] \mathrm{DG}$ concentrations, plasma was deproteinized with $\mathrm{ZnSO}_{4}$ and $\mathrm{Ba}(\mathrm{OH})_{2}$, dried to remove ${ }^{3} \mathrm{H}_{2} \mathrm{O}$, resuspended in water, and counted in scintillation fluid (Ultima Gold; Packard Instrument Co., Meriden, Connecticut, USA) on dual channels for separation of ${ }^{3} \mathrm{H}$ and ${ }^{14} \mathrm{C}$. The plasma concentration of ${ }^{3} \mathrm{H}_{2} \mathrm{O}$ was determined by the difference between ${ }^{3} \mathrm{H}$ counts without and with drying. For the determination of tissue 2-[ $\left.{ }^{14} \mathrm{C}\right] \mathrm{DG}-6$-phosphate (2-DG-6-P) content, tissue samples were homogenized, and the supernatants were subjected to an ion-exchange column to separate 2-DG-6-P from 2-DG, as described previously (15). The radioactivity of ${ }^{3} \mathrm{H}$ in tissue glycogen was determined by digesting tissue samples in $\mathrm{KOH}$ and precipitating glycogen with ethanol as described previously (16).

Calculations. Rates of basal glucose turnover and whole body glucose uptake were determined as the ratio of the $\left[{ }^{3} \mathrm{H}\right]$ glucose infusion rate (disintegrations per minute per minute) to the specific activity of plasma glucose (disintegrations per minute per micromole) at the end of the basal period and during the final 30 minutes of clamps, respectively. Hepatic glucose production (HGP) during the hyperinsulinemic-euglycemic clamps was determined by subtracting the glucose infusion rate from the whole body glucose uptake. Whole body glycolysis was calculated from the rate of increase in plasma ${ }^{3} \mathrm{H}_{2} \mathrm{O}$ concentration, determined by linear regression of the measurements at $80,90,100,110$, and 120 minutes. Whole body glycogen and lipid synthesis were estimated by subtracting whole body glycolysis from whole body glucose uptake, assuming that glycolysis and glycogen/lipid synthesis account for the majority of insulin-stimulated glucose uptake (17). Glucose uptake in individual tissues was calculated from plasma $2-\left[{ }^{14} \mathrm{C}\right] D G$ profile, which was fitted with a double exponential curve using MLAB (Civilized Software, Bethesda, Maryland, USA) and tissue 2-DG-6-P content as described previously (18). Skeletal muscle glycogen synthesis was calculated from ${ }^{3} \mathrm{H}$ incorporation to muscle glycogen as described previously (18). Skeletal muscle glycolysis was then estimated as the difference between muscle glucose uptake and muscle glycogen synthesis.

Statistical analysis. Data are expressed as means plus 
Table 1

Metabolic parameters during basal and hyperinsulinemic-euglycemic clamp periods in the control, heterozygous KO, muscle GLUT4 KO, and PHZ-treated muscle GLUT4 KO mice at 20-21 weeks of age and in the young control and young muscle GLUT4 KO mice at approximately 10 weeks of age

\begin{tabular}{|c|c|c|c|c|c|c|c|c|c|}
\hline & & & & \multicolumn{3}{|c|}{ Basal period } & \multicolumn{3}{|c|}{ Clamp period } \\
\hline & & $n$ & $\begin{array}{l}\text { Age } \\
(w k s)\end{array}$ & $\begin{array}{l}\text { Body } \\
\text { weight } \\
\text { (g) }\end{array}$ & $\begin{array}{c}\text { Plasma } \\
\text { glucose } \\
(\mathrm{mM})\end{array}$ & $\begin{array}{c}\text { Plasma } \\
\text { insulin } \\
(\mathrm{pM})\end{array}$ & $\begin{array}{c}\text { Plasma } \\
\text { glucose } \\
(\mathrm{mM})\end{array}$ & $\begin{array}{c}\text { Plasma } \\
\text { insulin } \\
(\mathrm{pM})\end{array}$ & $\begin{array}{c}\text { Glucose } \\
\text { infusion } \\
(\mu \mathrm{mol} / \mathrm{kg} / \mathrm{min})\end{array}$ \\
\hline Control & $\begin{array}{l}\text { Wild-type } \\
\text { lox/lox } \\
\text { cre }\end{array}$ & $\begin{array}{c}5 \\
10 \\
8\end{array}$ & $\begin{array}{l}21 \pm 1 \\
21 \pm 1 \\
21 \pm 1\end{array}$ & $\begin{array}{l}29 \pm 1 \\
31 \pm 2 \\
33 \pm 1\end{array}$ & $\begin{array}{l}6.8 \pm 0.1 \\
6.8 \pm 0.5 \\
7.2 \pm 0.4\end{array}$ & $\begin{array}{r}101 \pm 18 \\
78 \pm 12 \\
75 \pm 12\end{array}$ & $\begin{array}{l}6.3 \pm 0.1 \\
6.4 \pm 0.5 \\
6.2 \pm 0.4\end{array}$ & $\begin{array}{l}730 \pm 45 \\
728 \pm 64 \\
731 \pm 47\end{array}$ & $\begin{array}{l}197 \pm 4 \\
206 \pm 19 \\
202 \pm 11\end{array}$ \\
\hline Het KO & cre $+/$ lox & 8 & $21 \pm 1$ & $34 \pm 1$ & $7.3 \pm 0.4$ & $115 \pm 24$ & $7.1 \pm 0.3$ & $815 \pm 51$ & $143 \pm 9^{A}$ \\
\hline \multicolumn{2}{|c|}{ Muscle GLUT4 KO } & 8 & $21 \pm 1$ & $34 \pm 2$ & $8.2 \pm 0.4^{\mathrm{A}}$ & $86 \pm 21$ & $7.1 \pm 0.4$ & $781 \pm 61$ & $59 \pm 16^{A}$ \\
\hline \multicolumn{2}{|c|}{ PHZ-treated MG4KO } & 5 & $20 \pm 1$ & $27 \pm 1$ & $7.0 \pm 0.7$ & $166 \pm 30^{A, B}$ & $7.4 \pm 1.1$ & $638 \pm 60$ & $133 \pm 8^{\mathrm{A}, \mathrm{B}}$ \\
\hline \multicolumn{2}{|c|}{$\begin{array}{l}\text { Young control } \\
\text { Young MG4KO }\end{array}$} & $\begin{array}{c}9 \\
11\end{array}$ & $\begin{array}{l}10 \pm 0 \\
10 \pm 0\end{array}$ & $\begin{array}{l}31 \pm 1 \\
28 \pm 1\end{array}$ & $\begin{array}{l}7.1 \pm 0.5 \\
7.2 \pm 0.6\end{array}$ & $\begin{array}{c}53 \pm 4 \\
110 \pm 13 c\end{array}$ & $\begin{array}{l}6.9 \pm 0.6 \\
7.4 \pm 0.6\end{array}$ & $\begin{array}{l}618 \pm 49 \\
691 \pm 96\end{array}$ & $\begin{array}{r}211 \pm 13 \\
75 \pm 1^{C}\end{array}$ \\
\hline
\end{tabular}

${ }^{A} P<0.05$ vs. control mice by Duncan's multiple range test. ${ }^{B} P<0.05$ for PHZ-treated muscle GLUT4 KO mice vs. nontreated muscle GLUT4 KO mice by Duncan's multiple range test. ${ }^{C} P<0.05$ for young muscle GLUT4 KO mice vs. young control mice by the unpaired Student's $t$ test.

or minus SE. The significance of the difference in mean values among control, heterozygous $\mathrm{KO}$, muscle GLUT4 KO, and PHZ-treated muscle GLUT4 KO groups was evaluated using the Duncan's multiple range test. The significance of the difference in mean values between young control and young muscle GLUT4 KO groups was evaluated using the unpaired Student's $t$ test.

\section{Results}

The metabolic consequences of decreasing muscle GLUT4 content were examined during a 2-hour hyperinsulinemic-euglycemic clamp in awake 21-week-old control (wild-type, lox/lox, and cre), heterozygous KO, muscle GLUT4 KO, PHZ-treated muscle GLUT4 KO, and in 10-week-old young muscle GLUT4 KO mice. Both mice expressing cre without lox (cre) and mice homozygous for lox but not expressing cre (lox/lox) were studied as controls. There were no differences in any metabolic parameters between wild-type, lox/lox, and cre (Table 1), and results from these three control groups were combined for the figures. Basal plasma glucose concentration was increased by approximately $20 \%$ in the muscle GLUT4 KO mice compared with the controls $(P<0.05)$ (Table 1$)$, and PHZ treatment of muscle GLUT4 KO mice prevented the increase in basal plasma glucose concentration (Figure 1a). In contrast, basal plasma glucose concentration was not different in the young muscle GLUT4 KO mice compared with the young control mice (Table 1). Basal plasma insulin concentration following an overnight fast was not different in the 21-week-old muscle GLUT4 KO mice compared with the controls (Table 1), whereas it was significantly increased in the PHZ-treated muscle GLUT4 KO and young muscle GLUT4 KO mice compared with the respective controls $(P<0.05$ for both) (Figure 1b). During the clamps, plasma insulin concentrations were raised to approximately $720 \mathrm{pM}$, while plasma glucose was clamped at approximately $6.8 \mathrm{mM}$ in all groups (Table 1). The rate of glucose infusion

\section{Figure 1}

Whole body and skeletal muscle metabolic parameters during hyperinsulinemic-euglycemic clamps in awake mice at 20-21 weeks of age. (a) Basal plasma glucose concentration in the control (wild-type, lox/lox, and cre; open circles), muscle GLUT4 KO (MG4KO; filled circles), and PHZ-treated muscle GLUT4 KO (MG4KO + PHZ; open squares) mice. (b) Basal plasma insulin concentration in the control (open circles), muscle GLUT4 KO (filled circles), and PHZ-treated muscle GLUT4 $\mathrm{KO}$ (open squares) mice. (c) Insulin-stimulated whole body glucose uptake in vivo in control (open bar), heterozygous $\mathrm{KO}$ (Het KO; dark gray bar), muscle GLUT4 KO (black bar), and PHZ-treated muscle GLUT4 KO (MG4KO + PHZ; light gray bar) mice. (d) Insulin-stimulated glucose uptake in skeletal muscle (gastrocnemius) in vivo in control (open bar), heterozygous KO (dark gray bar), muscle GLUT4 KO (black bar), and PHZ-treated muscle GLUT4 KO (light gray bar) mice. Values are means plus or minus SE for 5-10 experiments. ${ }^{*} P<0.05$ vs. control mice; ${ }^{\#} P<0.05$ for the PHZ-treated muscle GLUT4 $\mathrm{KO}$ mice vs. nontreated muscle GLUT4 KO mice.
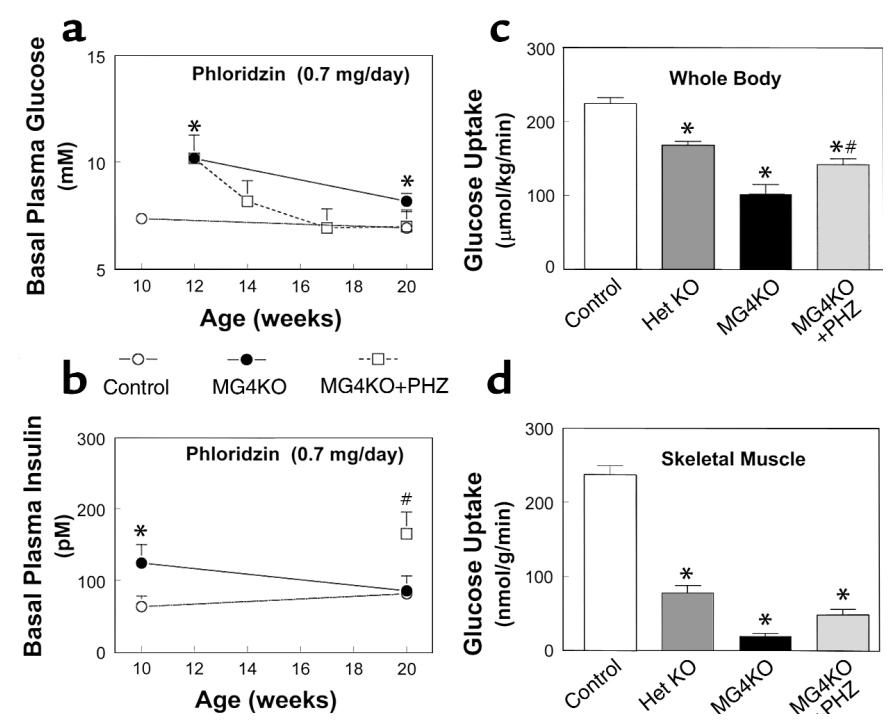


\section{Figure 2}

Insulin-stimulated glucose metabolic flux in whole body and skeletal muscle (gastrocnemius) during hyperinsulinemic-euglycemic clamps in awake control (wild-type, lox/lox, and cre; open bar), heterozygous $\mathrm{KO}$ (dark gray bar), muscle GLUT4 KO (black bar), and PHZ-treated muscle GLUT4 KO (light gray bar) mice at 20-21 weeks of age. (a) Insulin-stimulated whole body glycolysis in vivo. (b) Insulin-stimulated whole body glycogen/lipid synthesis in vivo. (c) Insulin-stimulated skeletal muscle glycolysis in vivo. (d) Insulin-stimulated skeletal muscle glycogen synthesis in vivo. Values are means plus or minus SE for $5-10$ experiments. ${ }^{*} P<0.05$ vs. control mice; ${ }^{P} P<0.05$ for the PHZ-treated muscle GLUT4 KO mice vs. nontreated muscle GLUT4 KO mice.

needed to maintain euglycemia increased rapidly in the control mice and reached a steady state within 90 minutes. In contrast, the glucose infusion rates in response to insulin were markedly reduced in muscle GLUT4 KO, young muscle GLUT4 KO, PHZ-treated muscle GLUT4 KO, and heterozygous KO mice $(P<0.005$ for all) (Table1).

Insulin-stimulated whole body glucose uptake was significantly decreased by $55 \%$ in the muscle GLUT 4 KO mice $(102 \pm 13$ vs. $224 \pm 8 \mu \mathrm{mol} / \mathrm{kg} / \mathrm{min}$ in controls; $P<$ $0.001)$ indicating severe insulin resistance in these mice (Figure 1c). Insulin-stimulated whole body glucose uptake was also reduced in the heterozygous $\mathrm{KO}$ mice $(P<0.001)$. Insulin-stimulated glucose uptake in skeletal muscle (gastrocnemius) was decreased by $92 \%$ in the muscle GLUT4 KO mice $(20 \pm 4$ vs. $237 \pm 12$ $\mathrm{nmol} / \mathrm{g} / \mathrm{min}$ in controls; $P<0.001$ ) (Figure $1 \mathrm{~d}$ ). Thus, the decrease in insulin-stimulated whole body glucose uptake could be attributed to a decrease in insulin-stimulated glucose uptake in skeletal muscle. Insulin-stimulated muscle glucose uptake was also reduced in the heterozygous $\mathrm{KO}$ mice $(P<0.001)$, and this result is consistent with the fact that heterozygous KO mice have a $40-50 \%$ reduction of muscle GLUT4 content compared with the controls (12). Insulin-stimulated whole body

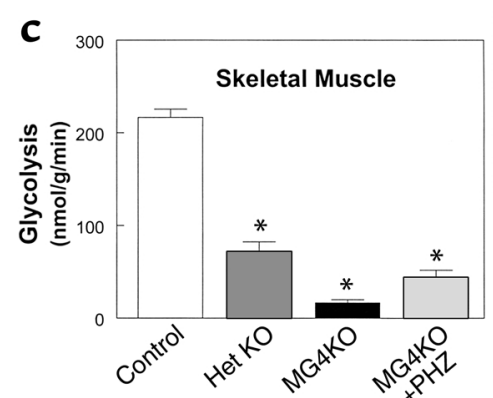

d

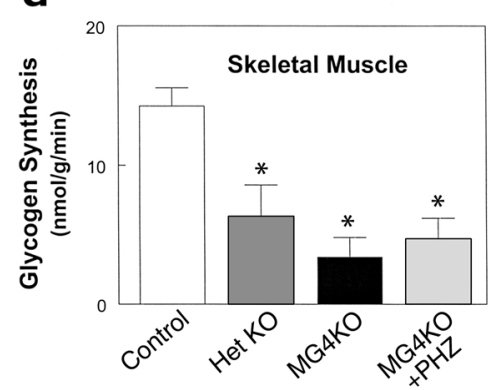

glycolysis and glycogen/lipid synthesis were decreased by $59 \%$ ( $49 \pm 8$ vs. $118 \pm 8 \mu \mathrm{mol} / \mathrm{kg} / \mathrm{min}$ in controls; $P<$ $0.001)$ and $50 \%(53 \pm 10$ vs. $106 \pm 9 \mu \mathrm{mol} / \mathrm{kg} / \mathrm{min}$ in controls; $P<0.001)$, respectively, in the muscle GLUT4 $\mathrm{KO}$ mice (Figure 2, a and b). Similar to the changes in whole body glucose metabolism, insulin-stimulated muscle glycolysis and glycogen synthesis were also decreased by $92 \%(17 \pm 3$ vs. $217 \pm 9 \mathrm{nmol} / \mathrm{g} / \mathrm{min}$ in controls; $P<0.001)$ and $76 \%(3 \pm 1 \mathrm{vs.} 14 \pm 1 \mathrm{nmol} / \mathrm{g} / \mathrm{min}$ in controls; $P<0.005)$, respectively, in the muscle GLUT4 KO mice (Figure 2, c and d). Surprisingly, insulin's ability to suppress HGP during clamps was significantly impaired in the muscle GLUT4 KO mice $(P<0.01)$ (Figure 3a). Moreover, insulin-stimulated glucose uptake in epididymal white adipose tissue and intrascapular brown adipose tissue were decreased by $69 \%$ ( $9 \pm 4$ vs. 28 $\pm 3 \mathrm{nmol} / \mathrm{g} / \mathrm{min}$ in controls; $P<0.001)$ and $84 \%(37 \pm 9$ vs. $237 \pm 24 \mathrm{nmol} / \mathrm{g} / \mathrm{min}$ in controls; $P<0.001)$, respectively, in the muscle GLUT4 KO mice (Figure 3, b and c).
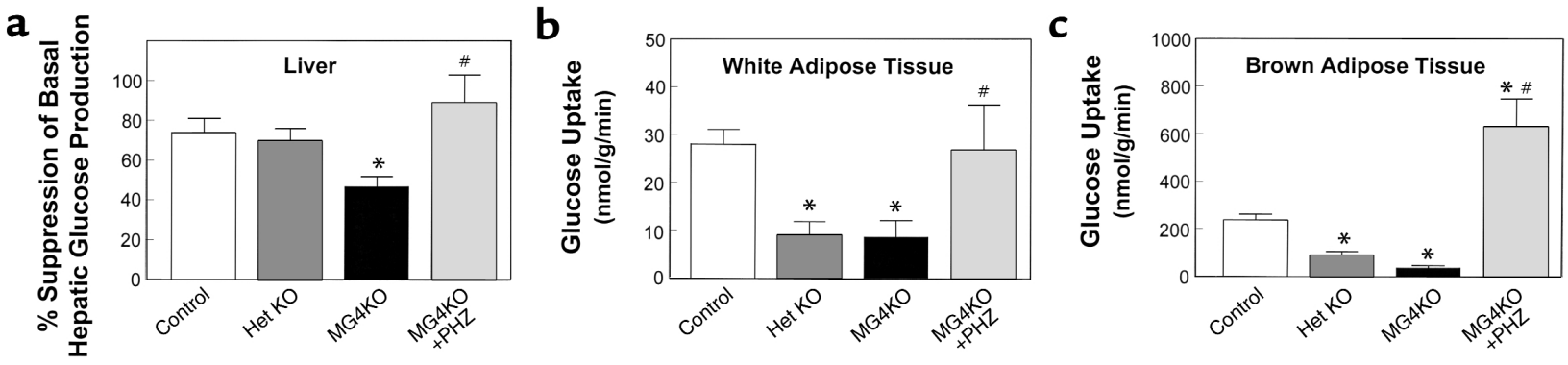

Figure 3

Insulin action in liver and adipose tissues during hyperinsulinemic-euglycemic clamps in awake control (wild-type, lox/lox, and cre; open bar), heterozygous KO (dark gray bar), muscle GLUT4 KO (black bar), and PHZ-treated muscle GLUT4 KO (light gray bar) mice at 20-21 weeks of age. (a) Percentage of suppression of basal hepatic glucose production. (b) Insulin-stimulated glucose uptake in epididymal white adipose tissue in vivo. (c) Insulin-stimulated glucose uptake in intrascapular brown adipose tissue in vivo. Values are means plus or minus SE for 5-10 experiments. ${ }^{*} P<0.05$ vs. control mice. ${ }^{\# P}<0.05$ for the PHZ-treated muscle GLUT4 KO mice vs. nontreated muscle GLUT4 KO mice. 

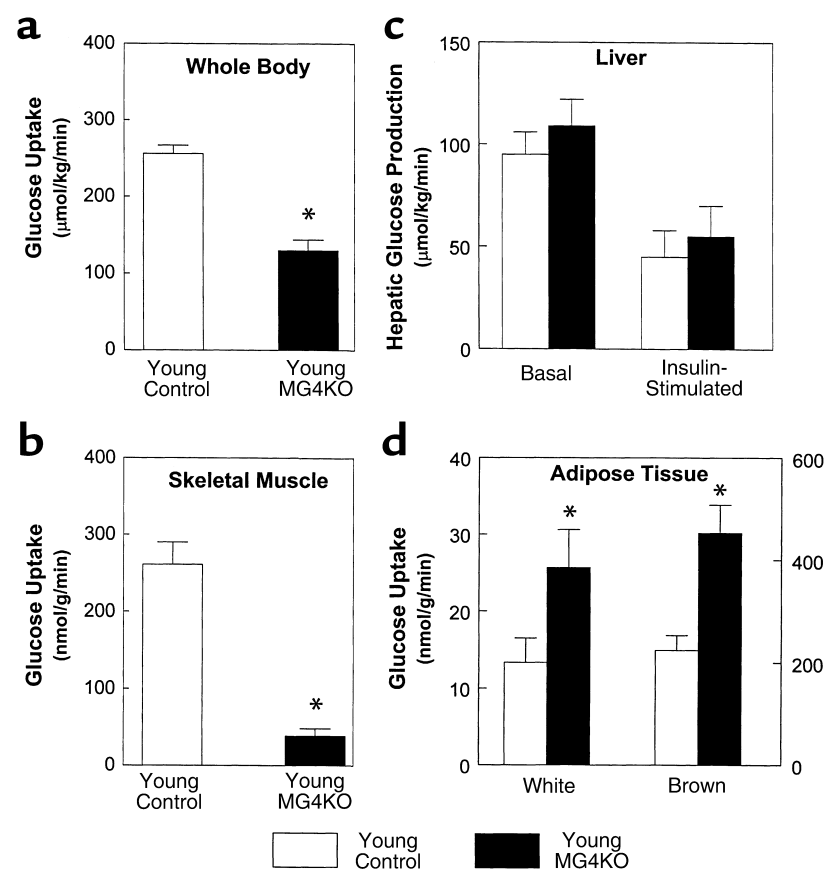

Figure 4

Insulin action in whole body and individual tissues during hyperinsulinemic-euglycemic clamps in awake young control (wild-type, lox/lox, and cre; open bar) and young muscle GLUT4 KO (MG4KO; filled bar) mice at approximately 10 weeks of age. (a) Insulin-stimulated whole body glucose uptake in vivo. (b) Insulin-stimulated glucose uptake in skeletal muscle (gastrocnemius) in vivo. (c) Basal and insulin-stimulated rates of hepatic glucose production. (d) Insulinstimulated glucose uptake in epididymal white adipose tissue and intrascapular brown adipose tissue in vivo. Values are means plus or minus SE for $9-11$ experiments. ${ }^{*} P<0.05$ vs. young control mice.

Insulin actions on whole body and individual tissues were also examined during a 2-hour hyperinsulinemic-euglycemic clamp in approximately 10 -week-old muscle GLUT4 KO mice. Insulin-stimulated whole body glucose uptake was significantly decreased by $49 \%$ in the young muscle GLUT4 KO mice $(130 \pm 14$ vs. $256 \pm 11 \mu \mathrm{mol} / \mathrm{kg} / \mathrm{min}$ in controls; $P<0.001)$, indicating severe insulin resistance in these mice (Figure 4a). Insulin-stimulated glucose uptake in skeletal muscle was decreased by $85 \%$ in the young muscle GLUT4 KO mice ( $39 \pm 10$ vs. $261 \pm 29 \mathrm{nmol} / \mathrm{g} / \mathrm{min}$ in controls; $P<0.001$ ) (Figure $4 \mathrm{~b}$ ), indicating that the decrease in insulin-stimulated whole body glucose uptake could be attributed to a decrease in insulinstimulated glucose uptake in skeletal muscle. Insulinstimulated whole body glycolysis and glycogen/lipid synthesis were decreased by $43 \%$ (95 \pm 15 vs. $166 \pm 21$ $\mu \mathrm{mol} / \mathrm{kg} / \mathrm{min}$ in controls; $P<0.01)$ and $60 \%$ (36 \pm 8 vs. $90 \pm 14 \mu \mathrm{mol} / \mathrm{kg} / \mathrm{min}$ in controls; $P<0.005$ ), respectively, in the young muscle GLUT4 KO mice (Figure 5, $a$ and $b$ ). Similar to the changes in whole body glucose metabolism, insulin-stimulated muscle glycolysis and glycogen synthesis were also decreased by $92 \%(18 \pm 3$ vs. $223 \pm 26 \mathrm{nmol} / \mathrm{g} / \mathrm{min}$ in controls; $P<0.001)$ and
$89 \%(0.7 \pm 0.2$ vs. $6.3 \pm 2.5 \mathrm{nmol} / \mathrm{g} / \mathrm{min}$ in controls; $P<0.05)$, respectively, in the young muscle GLUT4 KO mice (Figure 5, $\mathrm{c}$ and $\mathrm{d}$ ). These findings are consistent with glucose intolerance and insulin resistance as measured during glucose tolerance test and insulin tolerance test, respectively, in the 8-week-old muscle GLUT4 KO mice (12). In contrast, basal HGP and insulin's ability to suppress HGP were not changed in the young muscle GLUT4 KO mice (Figure 4c), suggesting that ablation of GLUT4 gene in skeletal muscle caused a selective muscle insulin resistance with normal hepatic insulin action in the young muscle GLUT4 KO mice. Interestingly, insulin-stimulated glucose uptake in white adipose tissue $(26 \pm 5$ vs. $13 \pm 3 \mathrm{nmol} / \mathrm{g} / \mathrm{min}$ in controls; $P<0.05)$ and brown adipose tissue ( $453 \pm 55$ vs. $224 \pm 29 \mathrm{nmol} / \mathrm{g} / \mathrm{min}$ in controls; $P<0.005)$ were increased twofold in the young muscle GLUT4 KO mice (Figure 4d).

To test the hypothesis that defects in insulin action in liver and adipose tissues of the muscle GLUT4 KO mice at 20-21 weeks of age are acquired and may be secondary to chronic hyperglycemia, we treated young muscle GLUT4 KO mice with PHZ from 12 weeks of age and assessed insulin action at 20 weeks of age. PHZ is an inhibitor of renal glucose reabsorption (13) and can be used to prevent hyperglycemia in the muscle GLUT4 KO mice. Basal plasma glucose concentration was significantly increased at the beginning of $\mathrm{PHZ}$

a

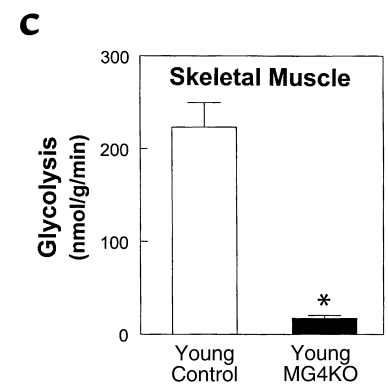

b
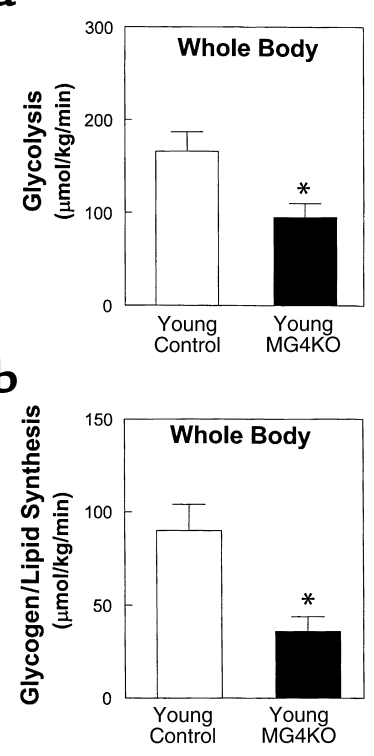

d

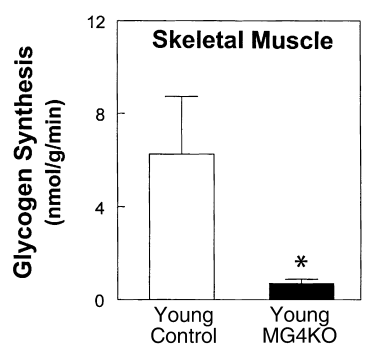

Figure 5

Insulin-stimulated glucose metabolic flux in whole body and skeletal muscle (gastrocnemius) during hyperinsulinemic-euglycemic clamps in awake young control (wild-type, lox/lox, and cre; open bar) and young muscle GLUT4 KO (filled bar) mice at approximately 10 weeks of age. (a) Insulin-stimulated whole body glycolysis in vivo. (b) Insulinstimulated whole body glycogen/lipid synthesis in vivo. (c) Insulinstimulated skeletal muscle glycolysis in vivo. (d) Insulin-stimulated skeletal muscle glycogen synthesis in vivo. Values are means plus or minus SE for 9-11 experiments. ${ }^{*} P<0.05$ vs. young control mice. 
treatment (at 12 weeks of age) $(10.2 \pm 1.1$ vs. $6.7 \pm 0.8$ $\mathrm{mM}$ in controls; $P<0.05$ ) but gradually decreased to a value not significantly different from the control mice after PHZ treatment and at the time of in vivo study (at 20 weeks of age) $(8.2 \pm 1.0,6.9 \pm 0.9$, and $7.0 \pm 0.7 \mathrm{mM}$ in the PHZ-treated muscle GLUT4 KO mice at 14, 17, and 20 weeks of age, respectively, vs. $7.0 \pm 0.3 \mathrm{mM}$ in controls; $P>0.05$ ) (Figure 1a). Insulin-stimulated whole body glucose uptake was decreased by $32 \%$ (142 \pm 8 vs. $224 \pm 8 \mu \mathrm{mol} / \mathrm{kg} / \mathrm{min}$ in controls; $P<0.01$ ), while insulin-stimulated glucose uptake in skeletal muscle was decreased by $81 \%$ in the PHZ-treated muscle GLUT4 KO mice ( $49 \pm 8$ vs. $237 \pm 12 \mathrm{nmol} / \mathrm{g} / \mathrm{min}$ in controls; $P<0.001$ ) (Figure 1, c and d). Insulin-stimulated whole body glycolysis and glycogen/lipid synthesis were similarly decreased by $30 \%$ ( $98 \pm 7$ vs. $118 \pm 8$ $\mu \mathrm{mol} / \mathrm{kg} / \mathrm{min}$ in controls; $P<0.05)$ and $36 \%(44 \pm 8 \mathrm{vs}$. $106 \pm 9 \mu \mathrm{mol} / \mathrm{kg} / \mathrm{min}$ in controls; $P<0.05$ ), respectively, in the PHZ-treated muscle GLUT4 KO mice (Figure 2 , $a$ and b). Similar to the changes in whole body glucose metabolism, insulin-stimulated muscle glycolysis and glycogen synthesis were also decreased by $82 \%$ ( $44 \pm 8$ vs. $251 \pm 43 \mathrm{nmol} / \mathrm{g} / \mathrm{min}$ in controls; $P<0.005$ ) and $45 \%(4.7 \pm 1.5$ vs. $8.7 \pm 1.7 \mathrm{nmol} / \mathrm{g} / \mathrm{min}$ in controls; $P<0.05)$, respectively, in the PHZ-treated muscle GLUT4 KO mice (Figure 2, c and d). In contrast, insulin's ability to suppress HGP was not significantly different in the PHZ-treated muscle GLUT4 KO mice compared with the controls (Figure 3a). Interestingly and similar to the results of the young muscle GLUT4 $\mathrm{KO}$ mice, insulin-stimulated glucose uptake in brown adipose tissue was significantly increased in the PHZtreated muscle GLUT4 KO mice (633 \pm 114 vs. $237 \pm 24$ $\mathrm{nmol} / \mathrm{g} / \mathrm{min}$ in controls; $P<0.05$ ), while that in white adipose tissue was unchanged in the PHZ-treated muscle GLUT4 $\mathrm{KO}$ mice $(27 \pm 9$ vs. $28 \pm 3 \mathrm{nmol} / \mathrm{g} / \mathrm{min}$ in controls; $P>0.05$ ) (Figure 3, $\mathrm{b}$ and $\mathrm{c}$ ).

\section{Discussion}

Several previous studies have examined the role of muscle glucose transport in whole body glucose homeostasis using genetically engineered mice with whole body GLUT4 disruption and have shown conflicting results (19-22). Katz and colleagues (19) have shown that mice with homozygous disruption of whole body GLUT4 have normal fasting plasma glucose and insulin concentrations (at 2-4 months of age). Similarly, Rossetti and colleagues (20) have shown that mice with heterozygous disruption of whole body GLUT4 (at 4-5 months of age) have normal plasma glucose concentration despite a significant decrease in insulinstimulated muscle glucose transport. In contrast, Stenbit and colleagues (21) found that mice with heterozygous disruption of whole body GLUT4 develop a diabetes phenotype (e.g., hyperglycemia) associated with decreases in insulin-stimulated glucose transport in skeletal muscle. The reason for this discrepancy is unclear but may be due to a difference in the age of mice. In this regard, Stenbit and colleagues (22) have shown that $50-60 \%$ of heterozygous GLUT4 KO mice were normoglycemic at 2-4 months of age, whereas the majority became hyperglycemic at 5-10 months of age. The age-dependent changes in whole body glucose homeostasis is also evident in the present study where the young muscle-specific GLUT4 KO mice were normoglycemic at approximately 10 weeks of age but developed hyperglycemia at 20-21 weeks of age. Although Zisman and colleagues observed mild hyperglycemia at 8 weeks of age when mice were continuously housed in their original site (12), in this related cohort, which underwent shipping and surgery and a related mild weight loss, the plasma glucose concentration was normal at approximately 10 weeks of age.

Although Stenbit and colleagues (22) have shown that partial decreases in whole body GLUT4 content result in a diabetes phenotype, the relative contribution of reduced GLUT4 content in skeletal muscle versus that in adipose tissue is unclear. Shepherd and colleagues (23) have shown enhanced whole body glucose disposal in normal mice with high level of overexpression of GLUT4 selectively in adipose tissue. Tozzo and colleagues (24) have also shown that overexpression of GLUT4 in adipocytes increased insulin-stimulated whole body glucose disposal in mice made markedly hyperglycemic with streptozotocin. Furthermore, we have shown recently that mice with muscle-specific inactivation of the insulin receptor gene have relatively normal whole body glucose homeostasis (25) due to an increase in adipose tissue glucose uptake compensating for the significantly reduced insulin-stimulated muscle glucose uptake. These findings also suggest an important role of adipocytes (i.e., adipose tissue glucose transport) for whole body glucose homeostasis in the mouse.

To address the relative role and mechanism of muscle-specific insulin resistance on the development of diabetes, we examined the effect of muscle-specific inactivation of GLUT4 gene in insulin action. Muscle GLUT4 KO mice at 20-21 weeks of age have fasting hyperglycemia and are severely insulin-resistant, which may be mostly attributed to a significant decrease in insulin-stimulated glucose transport in skeletal muscle. These findings are consistent with the notion that skeletal muscle glucose transport plays a major role in the whole body glucose disposal $(4,5)$. On the other hand, this is in marked contrast to the findings in mice with muscle-specific inactivation of the insulin receptor gene, which had a normal fasting plasma glucose concentration despite significant decreases in insulin-stimulated muscle glucose uptake $(12,25)$. However, there are two notable differences in these two models of muscle-specific insulin resistance. First, decreases in insulin-stimulated muscle glucose uptake in the muscle GLUT4 KO mice ( $90 \%)$ were more severe than those in the muscle-specific insulin receptor knockout mice $(70 \%)$, which typically do not develop a diabetes phenotype (25). As a result, decreases in insulin-stimulated whole body glucose metabolism in the muscle GLUT4 KO mice were more severe 
than those in the muscle insulin receptor $\mathrm{KO}$ mice ( $\sim 55$ vs. $\sim 45 \%$ in muscle insulin-receptor KO mice). Second, the rates of basal glucose transport and contraction-stimulated glucose transport are also diminished in the muscle GLUT4 KO mice (12).

In addition to altered glucose transport, insulinstimulated glucose metabolic flux (i.e., glycolysis and glycogen synthesis) in skeletal muscle were similarly reduced in the muscle GLUT4 KO mice. Glucose transport is most likely rate controlling for glucose use in skeletal muscles $(9,26,27)$, and decreases in insulin-stimulated muscle glucose transport may account for the parallel decreases in glycolysis and glycogen synthesis in the muscle GLUT4 KO mice. Similar decreases in insulin-stimulated glucose transport and metabolic flux were observed in the musclespecific insulin receptor knockout mice (25); however, there was a noteworthy difference in the pattern of changes in glucose metabolic flux in these two insulin-resistant models of transgenic mice. The decrease in insulin-stimulated muscle glycogen synthesis was more profound than the decrease in muscle glucose uptake in muscle-specific insulin receptor knockout mice, which may be accounted for by combined defects in insulin's activation of glucose transport and glycogen synthase activity in these mice.

Despite decreases in insulin-stimulated muscle glucose uptake, insulin-stimulated glucose uptake in adipose tissue was significantly increased in the young muscle GLUT4 KO mice. This increase in adipose tissue glucose uptake may be a compensatory response to the reduced muscle glucose uptake in the young muscle GLUT4 KO mice (28). Interestingly, increases in insulin-stimulated adipose tissue glucose uptake were also observed in muscle-specific insulin receptor knockout mice that had a normal plasma glucose concentration and a mildly elevated plasma insulin concentration (25). Similarly, mild hyperinsulinemia may be responsible for increased insulin-stimulated adipose tissue glucose uptake in the young muscle GLUT4 KO mice that is consistent with previous studies showing that chronic hyperinsulinemia increased levels of GLUT4 and insulin-stimulated glucose transport in adipose tissue in rats $(29,30)$. This mild hyperinsulinemia in the young muscle GLUT4 KO mice may reflect a compensatory response of pancreatic $\beta$ cells to the severe muscle insulin resistance present in these mice. In this regard, normal plasma insulin concentrations, as compared with the control mice, despite mild hyperglycemia, suggest $\beta$ cell decompensation in the muscle GLUT4 KO mice at 20-21 weeks of age. Moreover, basal plasma insulin concentration in the muscle GLUT4 KO mice after a PHZ treatment and that in the young muscle GLUT4 KO mice before the onset of hyperglycemia were significantly increased as compared with the respective control mice, suggesting that $\beta$ cell decompensation in the muscle GLUT4 KO mice was acquired and prevented with PHZ treatment. This finding further suggests that $\beta$ cell decompensa- tion in the muscle GLUT4 KO mice may partly be due to chronic hyperglycemia (31). Interestingly, musclespecific insulin-receptor knockout mice with approximately $45 \%$ reduction in insulin-stimulated whole body glucose metabolism remained mildly hyperinsulinemic without hyperglycemia with aging (32), as opposed to the muscle GLUT4 KO mice (20-21 weeks old) with approximately $55 \%$ reduction in insulinstimulated whole body glucose metabolism, which subsequently developed a diabetes phenotype. These findings suggest that there may be a critical threshold (45-55\% reduction in insulin-stimulated whole body glucose metabolism) in whole body insulin resistance, which can result in $\beta$ cell decompensation and subsequent development of a diabetes phenotype.

The most important finding from this study was that in addition to the selective muscle insulin resistance, we also observed a significant decrease in insulin-stimulated glucose uptake in white and brown adipose tissue, as well as hepatic insulin resistance in the muscle GLUT4 KO mice. Since adipose tissue GLUT4 content was not altered in these mice (12), the adipocyte defect was most likely due to defects in insulin signaling and/or insulin activation of glucose transport (i.e., impaired insulin-mediated GLUT4 translocation or fusion with plasma membrane). Since insulin-stimulated glucose uptake in adipose tissue was increased in the young muscle GLUT4 KO mice, this defect in adipose tissue glucose uptake in the muscle GLUT4 KO mice at 20-21 weeks of age may be acquired and secondary to prolonged, albeit mild, hyperglycemia (i.e., glucose toxicity) in these mice $(33,34)$. Moreover, insulin's ability to suppress HGP was also significantly impaired in the muscle GLUT4 KO mice, whereas young muscle GLUT4 KO mice had normal insulin action in liver. These findings suggest that selective and severe skeletal muscle insulin resistance in the muscle GLUT4 KO mice may cause secondary defects in insulin actions in liver and adipose tissue (33-35).

To determine whether defects in insulin's ability to stimulate adipose tissue glucose uptake and suppress HGP may be acquired defects associated with chronic hyperglycemia, we assessed insulin action in the muscle GLUT4 KO mice after long-term (8 weeks) PHZ treatment, which inhibits renal glucose reabsorption (13), to prevent hyperglycemia. Insulin-stimulated muscle glucose uptake was significantly decreased in the PHZ-treated muscle GLUT4 KO mice, as observed in the young muscle GLUT4 KO mice and muscle GLUT4 KO mice at 20-21 weeks of age. In contrast, insulin-stimulated glucose uptake was significantly increased in the brown adipose tissue and was unchanged in the white adipose tissue in the PHZtreated muscle GLUT4 KO mice. Furthermore, insulin's ability to suppress HGP was normal in the PHZ-treated muscle GLUT4 KO mice. These results demonstrate that chronic $\mathrm{PHZ}$ treatment prevented the development of insulin resistance in adipose tissue 
and liver in the muscle GLUT4 KO mice and suggest that these defects may be acquired and secondary to prolonged hyperglycemia (31,33-35).

In summary, muscle-specific inactivation of the GLUT4 gene resulted in reduced insulin-stimulated glucose uptake in skeletal muscle and severe whole body insulin resistance due to additional acquired defects in insulin action in liver and adipose tissue. This is the first demonstration that a primary defect in insulin-stimulated muscle glucose uptake can cause insulin resistance in other insulin-responsive organs (liver and adipocytes) and subsequently result in the development of a diabetes phenotype. Furthermore, these acquired abnormalities in insulin action in liver and adipose tissue were prevented by $\mathrm{PHZ}$ treatment, which suggests that glucose toxicity was the responsible factor.

\section{Acknowledgments}

Our study was supported by grants from the United States Public Health Service: R01 DK-40936, P30 DK45735, and U24 DK-59635 (G.I. Shulman); R01 DK33201 (C.R. Kahn); and R01 DK-43051 (B.B. Kahn). J.K. Kim is a research associate, and G.I. Shulman is an Investigator of the Howard Hughes Medical Institute. We are grateful to Ying Zhu, Aida Groszmann, and Takamasa Higashimori for technical assistance.

1. Warram,J.H., Martin, B.C., Krolewski, A.S., Soeldner, J.S., and Kahn, C.R. 1990. Slow glucose removal rate and hyperinsulinemia precede the development of type II diabetes in the offspring of diabetic patients. Ann. Intern. Med. 113:909-915.

2. Reaven, G.M., Bernstein, R., Davis, B., and Olefsky, J.M. 1976. Nonketotic diabetes mellitus: insulin deficiency or insulin resistance? Am. J. Med. 60:80-88.

3. DeFronzo, R.A. 1988. The triumvirate: beta-cell, muscle, liver. A collusion responsible for NIDDM. Diabetes. 37:667-687.

4. Shulman, G.I., et al. 1990. Quantitation of muscle glycogen synthesis in normal subjects and subjects with non-insulin-dependent diabetes by ${ }^{13} \mathrm{C}$ nuclear magnetic resonance spectroscopy. N. Engl. J. Med. 322:223-228.

5. DeFronzo, R.A., et al. 1981. The effect of insulin on the disposal of intravenous glucose. Results from indirect calorimetry and hepatic and femoral venous catheterization. Diabetes. 30:1000-1007.

6. Shepherd, P.R., and Kahn, B.B. 1999. Glucose transporters and insulin action: implications for insulin resistance and diabetes mellitus. N. Engl. J. Med. 341:248-257.

7. Klip, A., Ramlal, T., Young, D.A., and Holloszy, J.O. 1987. Insulin-induced translocation of glucose transporters in rat hindlimb muscles. FEBS Lett. 224:224-230.

8. Kahn, B.B. 1996. Lilly lecture 1995. Glucose transport: pivotal step in insulin action. Diabetes. 45:1644-1654.

9. Cline, G.W., et al. 1999. Impaired glucose transport as a cause of decreased insulin-stimulated muscle glycogen synthesis in type 2 diabetes. N. Engl. J. Med. 341:240-246.

10. Rothman, D., et al. 1995. Decreased muscle glucose transport/phosphorylation is an early defect in the pathogenesis of non-insulin-dependent diabetes mellitus. Proc. Natl. Acad. Sci. USA. 92:983-987.

11. Petersen, K.F., et al. $1998 .{ }^{13} \mathrm{C} /{ }^{31} \mathrm{P}$ NMR studies on the mechanism of insulin resistance in obesity. Diabetes. 47:381-386.

12. Zisman, A., et al. 2000. Targeted disruption of the glucose transporter 4 selectively in muscle causes insulin resistance and glucose intolerance.
Nat.Med. 6:924-928.

13. Dimitrakoudis, D., Vranic, M., and Klip, A. 1992. Effects of hyperglycemia on glucose transporters of the muscle: use of the renal glucose reabsorption inhibitor phloridzin to control glycemia. J. Am. Soc. Nephrol. 3:1078-1091.

14. Kim, J.K., Gavrilova, O., Chen, Y., Reitman, M.L., and Shulman, G.I. 2000 Mechanism of insulin resistance in A-ZIP/F-1 fatless mice. J. Biol. Chem. 275:8456-8460.

15. Ohshima, K., Shargill, N.S., Chan, T.M., and Bray, G.A. 1984. Adrenalectomy reverses insulin resistance in muscle from obese (ob/ob) mice. $A m$. J. Physiol. 246:E193-E197.

16. Kim, J.K., Wi, J.K., and Youn, J.H. 1996. Plasma free fatty acids decrease insulin-stimulated skeletal muscle glucose uptake by suppressing glycolysis in conscious rats. Diabetes. 45:446-453.

17. Rossetti, L., and Giaccari, A. 1990. Relative contribution of glycogen synthesis and glycolysis to insulin-mediated glucose uptake. J. Clin. Invest. 85:1785-1792.

18. Youn, J.H., and Buchanan, T.A. 1993. Fasting does not impair insulinstimulated glucose uptake but alters intracellular glucose metabolism in conscious rats. Diabetes. 42:757-763.

19. Katz, E.B., Stenbit, A.E., Hatton, K., DePinho, R., and Charron, M.J. 1995. Cardiac and adipose tissue abnormalities but not diabetes in mice deficient in GLUT4. Nature. 377:151-155.

20. Rossetti, L., et al. 1997. Peripheral but not hepatic insulin resistance in mice with one disrupted allele of the glucose transporter type 4 (GLUT4) gene. J. Clin. Invest. 100:1831-1839.

21. Stenbit, A.E., et al. 1997. GLUT4 heterozygous knockout mice develop muscle insulin resistance and diabetes. Nat. Med. 3:1096-1101.

22. Stenbit, A.E., et al. 1996. Diverse effects of GLUT4 ablation on glucose uptake and glycogen synthesis in red and white skeletal muscle. J. Clin. Invest. 98:629-634.

23. Shepherd, P.R., et al. 1993. Adipose cell hyperplasia and enhanced glucose disposal in transgenic mice overexpressing GLUT4 selectively in adipose tissue. J. Biol. Chem. 268:2224-22246.

24. Tozzo, E., Gnudi, L., and Kahn, B.B. 1997. Amelioration of insulin resistance in streptozotocin diabetic mice by transgenic overexpression of GLUT4 driven by an adipose-specific promoter. Endocrinology. 138:1604-1611.

25. Kim, J.K., et al. 2000. Redistribution of substrates to adipose tissue in mice with selective insulin resistance in muscle promotes obesity. J. Clin. Invest. 105:1791-1797.

26. Ren, J.M., et al. 1993. Evidence from transgenic mice that glucose transport is rate-limiting for glycogen deposition and glycolysis in skeletal muscle. J. Biol. Chem. 268:16113-16115.

27. Ziel, F.H., Venkatesan, N., and Davidson, M.B. 1988. Glucose transport is rate limiting for skeletal muscle glucose metabolism in normal and STZ-induced diabetic rats. Diabetes. 37:885-890.

28. Laybutt, D.R., Chisholm, D.J., and Kraegen, E.W. 1997. Specific adaptations in muscle and adipose tissue in response to chronic systemic glucose oversupply in rats. Am. J. Physiol. 273:E1-E9.

29. Cusin, I., Terrettaz, J., Rohner-Jeanrenaud, F., and Jeanrenaud, B. 1990. Metabolic consequences of hyperinsulinemia imposed on normal rats on glucose handling by white adipose tissue, muscles, and liver. Biochem. J. 267:99-103.

30. Cusin, I., et al. 1990. Hyperinsulinemia increases the amount of GLUT4 mRNA in white adipose tissue and decreases that of muscles, a clue for increased fat depot and insulin resistance. Endocrinology. 127:3246-3248.

31. Boden, G., Ruiz, J., Kim, C.J., and Chen, X. 1996. Effects of prolonged glucose infusion on insulin secretion, clearance, and action in normal subjects. Am. J. Physiol. 270:E251-E258.

32. Bruning, J.C., et al. 1998. A muscle-specific insulin receptor knockout exhibits features of the metabolic syndrome of NIDDM without altering glucose tolerance. Mol. Cell. 2:559-569.

33. Rossetti, L. 1995. Glucose toxicity: the implications of hyperglycemia in the pathophysiology of diabetes mellitus. Clin. Invest. Med. 18:255-260.

34. Yki-Jarvinen, H., et al. 1997. UDP-N-acetylglucosamine transferase and glutamine: fructose 6-phosphate amidotransferase activities in insulinsensitive tissues. Diabetologia. 40:76-81.

35. Brichard, S.M., Henquin, J.C., and Girard, J. 1993. Phlorizin treatment of diabetic rats partially reverses the abnormal expression of genes involved in hepatic glucose metabolism. Diabetologia. 36:292-298. 